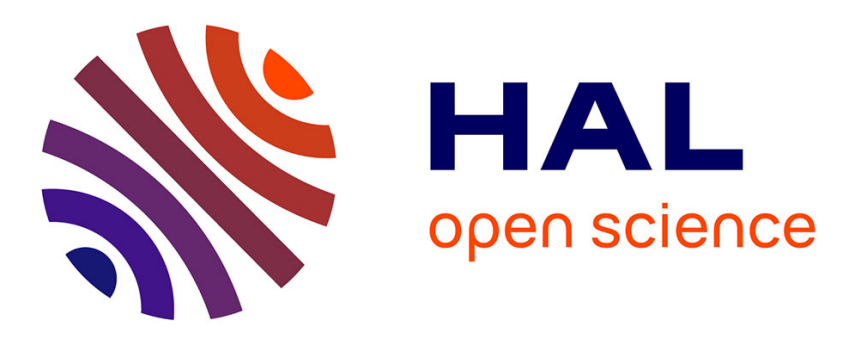

\title{
Prosthetic clone and natural human tooth comparison by speckle interferometry
}

Pierre Slangen, Stéphane Corn, Michel Fages, Jacques Raynal, Frédéric

Cuisinier

\section{- To cite this version:}

Pierre Slangen, Stéphane Corn, Michel Fages, Jacques Raynal, Frédéric Cuisinier. Prosthetic clone and natural human tooth comparison by speckle interferometry. Speckle 2010, Sep 2010, Florianapolis, United States. pp.73870D, 10.1117/12.870748 . hal-02012192

\section{HAL Id: hal-02012192 https://hal.science/hal-02012192}

Submitted on 8 Jun 2021

HAL is a multi-disciplinary open access archive for the deposit and dissemination of scientific research documents, whether they are published or not. The documents may come from teaching and research institutions in France or abroad, or from public or private research centers.
L'archive ouverte pluridisciplinaire HAL, est destinée au dépôt et à la diffusion de documents scientifiques de niveau recherche, publiés ou non, émanant des établissements d'enseignement et de recherche français ou étrangers, des laboratoires publics ou privés. 


\title{
Prosthetic Clone and Natural Human Tooth Comparison by Speckle Interferometry
}

\author{
Pierre Slangen ${ }^{\mathbf{a}}$, Stephane Corn ${ }^{\mathbf{a}}$, Michel Fages ${ }^{\mathbf{b}}$, Jacques Raynal ${ }^{\mathbf{b}}$, Frederic J.G. Cuisinier ${ }^{\mathbf{b}}$ \\ ${ }^{a}$ ISR, LGEI, Ecole des Mines d'Alès, 6 Avenue de Clavieres F-30319 Ales Cedex France \\ ${ }^{b}$ Université Montpellier Sud de France, EA 4203545 Avenue Prof. Viala, F-34193 Montpellier \\ Cedex5 France
}

\begin{abstract}
New trends in dental prosthodontic interventions tend to preserve the maximum of "body" structure. With the evolution of CAD-CAM techniques, it is now possible to measure "in mouth" the remaining dental tissues. The prosthetic crown is then designed using this shape on which it will be glued on, and also by taking into account the contact surface of the opposite jaw tooth.

Several theories discuss on the glue thickness and formulation, but also on the way to evolve to a more biocompatible crown and also new biomechanical concepts. In order to validate these new concepts and materials, and to study the mechanical properties and mechanical integrity of the prosthesis, high resolution optical measurements of the deformations of the glue and the crown are needed. Samples are two intact premolars extracted for orthodontics reasons. The reference sample has no modifications on the tooth while the second sample tooth is shaped to receive a feldspathic ceramic monoblock crown which will be glued.

This crown was manufactured with a chairside CAD-CAM system from an intra-oral optical print. The software allows to realize a nearly perfect clone of the reference sample. The necessary space for the glue is also entered with ideal values. This duplication process yields to obtain two samples with identical anatomy for further processing. The glue joint thickness can also be modified if required.

The purpose is to compare the behaviour of a natural tooth and its prosthetic clone manufactured with «biomechanical» concepts. Vertical cut samples have been used to deal with planar object observation, and also to look "inside" the tooth.

We have developed a complete apparatus enabling the study of the compressive mechanical behaviour of the concerned tooth by speckle interferometry.

Because in plane displacements are of great interest for orthodontic measurements ${ }^{1}$, an optical fiber in-plane sensitive interferometer has been designed. The fibers are wrapped around piezoelectric transducers to perform " 4-buckets" phase shifting leading to phase variations during the compression test.

In-plane displacement fields from speckle interferometry already showed very interesting data concerning the mechanical behaviour of teeth : the dentine-enamel junction (DEJ) and the glue junction have been shown including their interfacing function. Mechanical action of the tooth surrounding medium will also be discussed.
\end{abstract}

Keywords: CAD-CAD clone, speckle interferometry, dentine enamel joint, prosthetic tooth crown

\section{INTRODUCTION}

New trends in dental prosthodontic interventions tend to reach biomimetic behaviour. Evolutions of CAD-CAM techniques enable to build ceramic prosthetic crowns and, above all, to set the cement joint thickness that links crown and remaining dental tissues. CAD is based on "in-mouth" optical print (i.e. shape on which the clone is glued and contact surface of the opposite jaw tooth). Prosthetic crown is then manufactured, using these parameters, from a feldspathic ceramic rod. In this study, the cloning process gives two samples with identical shape for further use: clone and tooth compression response are measured by speckle interferometry. 


\section{DESIGN AND GOALS}

Several theories ${ }^{1}$ discuss the glue thickness and its formulation, acting as a crucial interface that accommodates the different stresses applied to the prosthetic tooth. Moreover this biomechanical analysis tries to reproduce the natural behaviour of the dentine-enamel joint (DEJ). In order to validate these new concepts and materials, and to study the mechanical properties and the mechanical integrity of the prosthesis, high resolution optical measurements of the deformations of glue and crown are required ${ }^{2}$. In our case, samples are two intact premolars extracted for orthodontic reasons from the same patient. The reference sample is preserved while the second sample tooth is shaped to receive a feldspathic ceramic monoblock crown (Vita MarkII, Vita), clone of the reference tooth.

The crown has been made by prosthetic CAD-CAD system from optical prints directly realized in-mouth. The associated software enables the realization of the perfect clone of the tooth. The system is a "dentist" chairside system from Sirona: CEREC 3D.

The best spacing required to realize the glue joint between the crown and the tooth is also computed. In our case $100 \mu \mathrm{m}$ are left between the tooth and the crown and solely $40 \mu \mathrm{m}$ in the cervical junction zone. The copy process yields to two identical samples with the same crown anatomy: the natural tooth and the crown clone realized by CAD-CAM technique.

It is then possible to charge with the same loads two teeth with similar morphology but with different structures (natural and prosthetic) and so to compare their compressive behaviour.

Vertical cuttings have also been used to allow planar object observation, and also to appreciate the differential behaviours "inside" the tooth: the crown-glue-dentine joint for the clone and the DEJ for natural tooth (Fig.1). Recent works also consider the whole natural tooth but do not allow the visualization of the internal parts of the tooth ${ }^{3}$. For this study, a tooth compression test device and an optical fibers in-plane sensitive speckle interferometer have been developed. Samples are white powdered to diffuse laser light.
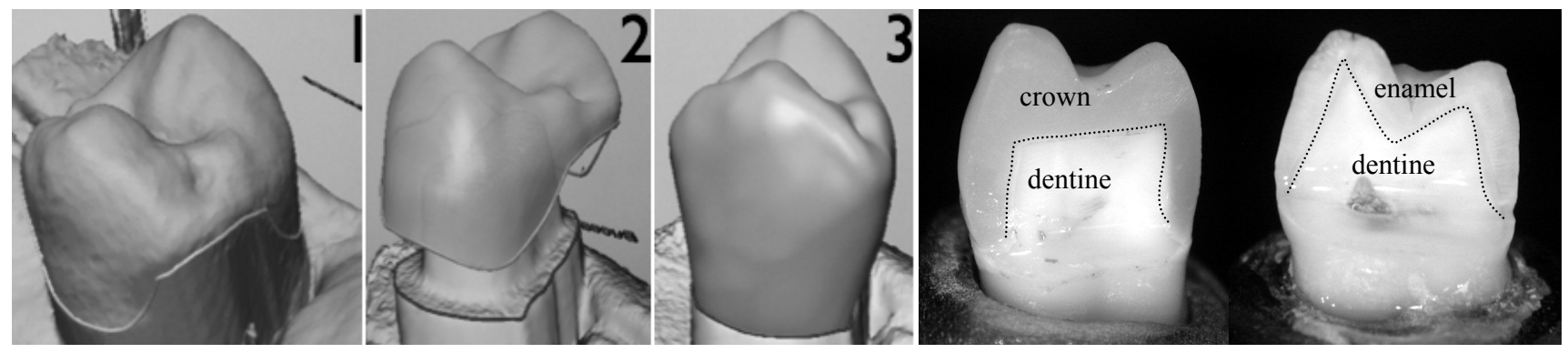

Fig. 1: CAD-CAM Cloning process in copy mode (1 ref optical print, 2 shaping, 3 CAM ready), clone and master cuts

\subsection{Mechanical set-up}

The mechanical set-up is presented in Fig.2. The compression test device fulfills the high sensitivity of speckle interferometry and also copes with the rigid body motions of the whole system. The sample tooth is placed in a dedicated mold in the lower jaw while the force transducer holder is slowly translated vertically by the stepping motor. The system can generate a „force-driven“ displacement, or just a „user“ displacement. The whole mechanical system is screwed on the holographic table top. Very small displacement steps, up to $1,6 \mathrm{~nm}$ can theoretically be achieved.

The force can be applied to the tooth directly with the force transducer or using a relay rod (shorter than shown Fig.2). We can point out that the force is always applied on the same part of the tooth surface for all the samples. This requires some degrees of adjustment to place the lower jaw properly. Samples have got molded jaw or have just been cemented.

The teeth are affixed to the lower jaw from the root by using a dedicated molded base. 


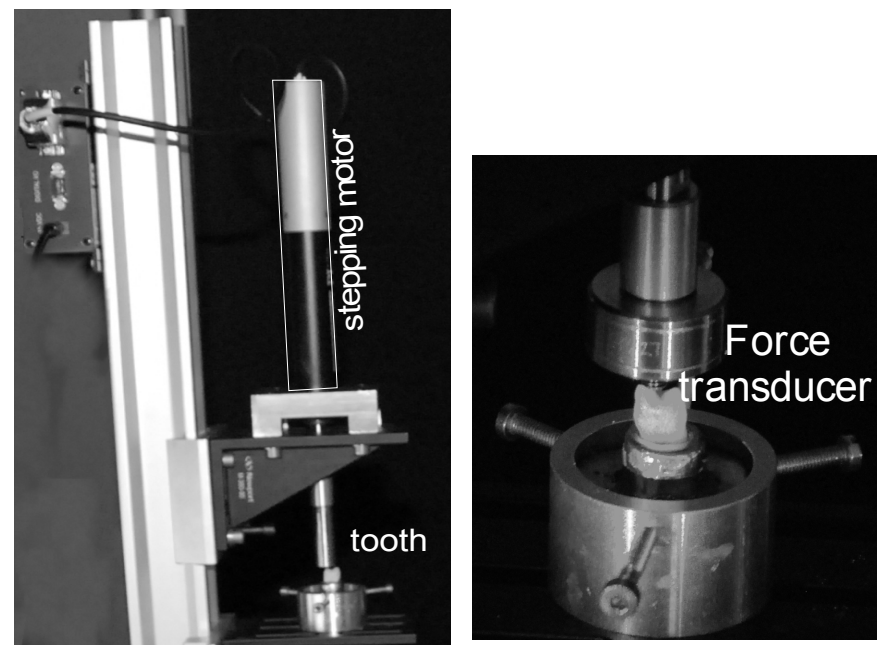

Fig. 2: Compression test mechanical set-up and dedicated molded lower jaw

\subsection{Optical set-up}

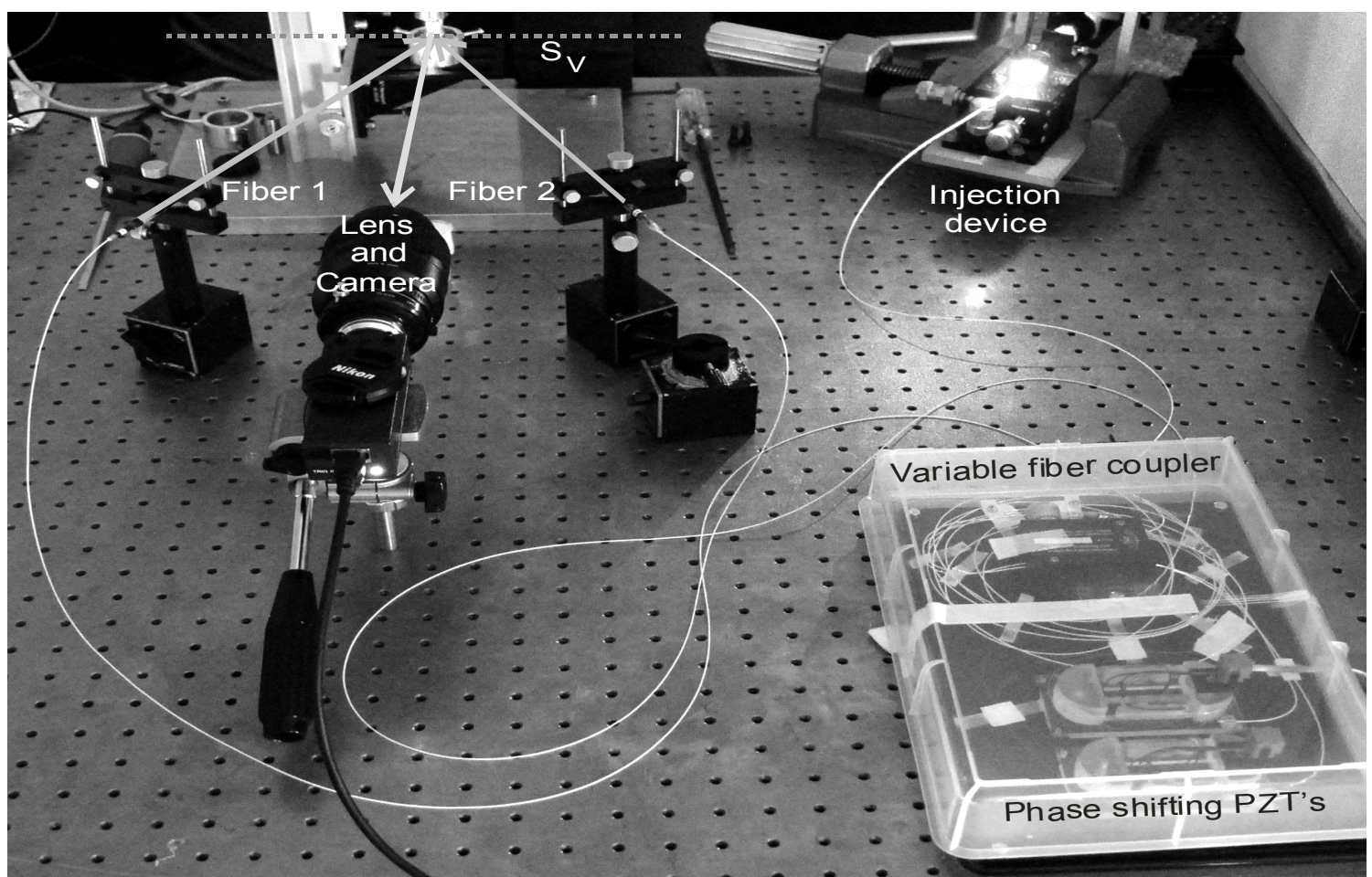

Fig. 3: In plane speckle interferometer, showing observation and illumination beams (full), sensitivity vector $\mathrm{S}_{\mathrm{V}}(\mathrm{dashed})$, variable fiber coupler, phase shifting and injection devices.

The optical set-up is presented in Fig.3. This COTS system (Canadian Instruments) offers injection, variable intensity coupling in the output fibers, and also phase shifting. There is two input fibres, one for injection and one for detection of the reflected signal at the output interfaces. Parts of the output fibers are bared and wrapped around piezoelectric transducers. The phase shift is applied on any or both of the two output fibers. The system is protected from thermal and mechanical effects by a plastic box and is easily breadboard able. Phase shifts have been calibrated using common procedures ${ }^{4}$. 
The results of calibration for the two piezoelectric transducers $d a 1$ and $d a 2$ are presented in table 1 . It is also possible to calibrate the system using the back reflections on the output fibres, producing fringes at the exit of the second input fibre (Table 2) and detected by a pin diode.

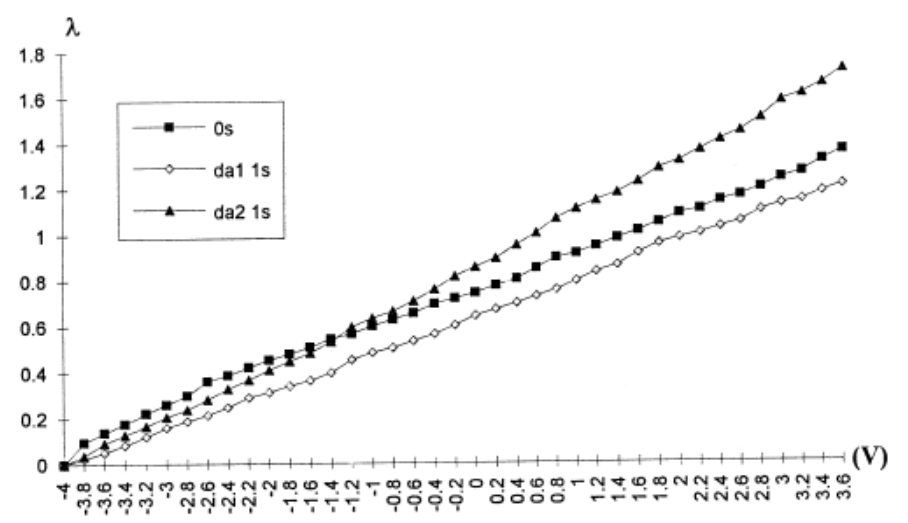

Table 1: Calibration curves of the PZT

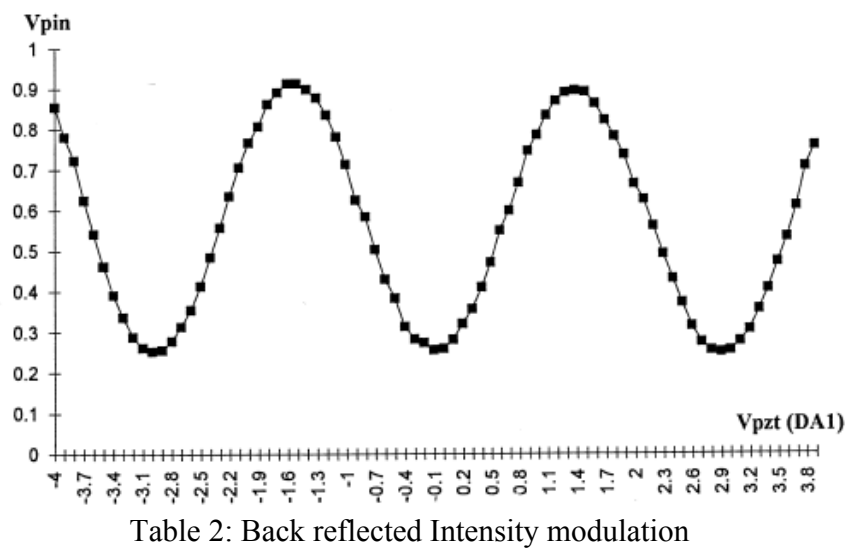

Because in-plane displacements are of great interest for orthodontic measurements, an optical fibers in-plane sensitive interferometer has been designed. The sensitivity vector, $\mathrm{S}_{\mathrm{v}}$, is showed at figure 3 . Horizontal sensitivity is achieved. A "4-buckets" phase shifting algorithm ${ }^{5}$ leads to phase variations during the compression test. The compression test consists of firstly increase the load until the whole set-up is well in place at about $120 \mathrm{~N}$. Then the force is decreased to the minimum contact and is finally slightly enhanced to generate live fringe maps. This is allowed thanks to the small increments of the step motor. Live fringes are displayed between a reference state and the current load state. Between minimal and maximal loading, different phase maps are recorded and stored in memory. Moreover the reference state is also refreshed as for some load steps the number of fringes can be very high and so the noise is too important to lead to good interpretation of the resulting fringes.

\section{RESULTS AND DISCUSSION}

First results have been obtained with samples cemented with araldite and for different loads (Fig.4). In-plane displacement fields from speckle interferometry already showed interesting data concerning the mechanical behaviour of the different tooth parts. The particular behaviour of the interface joining them histologically is very important for dental aspects. 

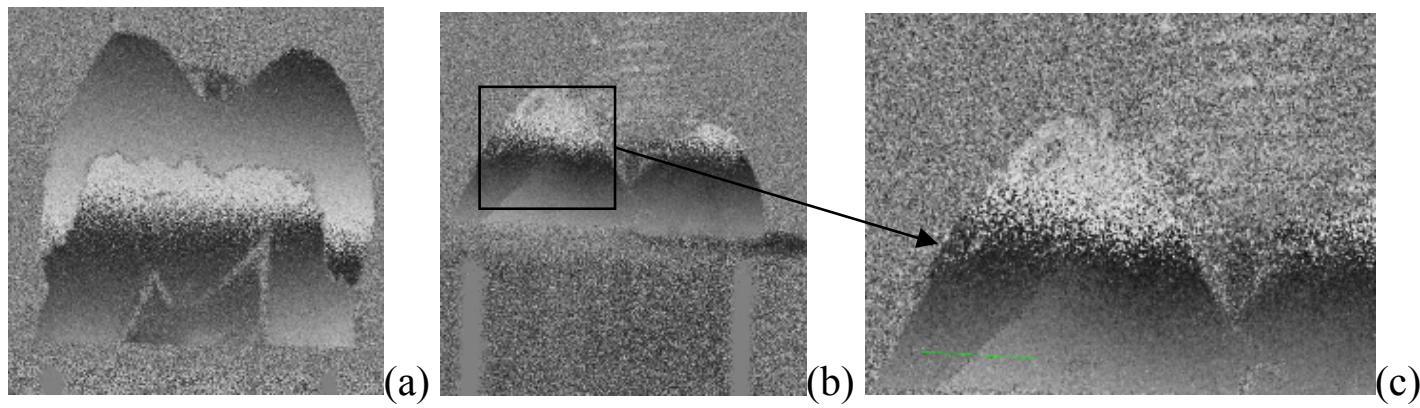

Fig. 4: Clone (a) and natural tooth (b) in-plane displacements, and (c) DEJ detection zoom

As the stabilization ramp has been applied, this shows off the samples have to be molded in the lower jaw by bulking it into chromium-cobalt mold obtained from lost wax technique. This ensures to cancel the effects of the glue between the tooth root and the lower jaw.
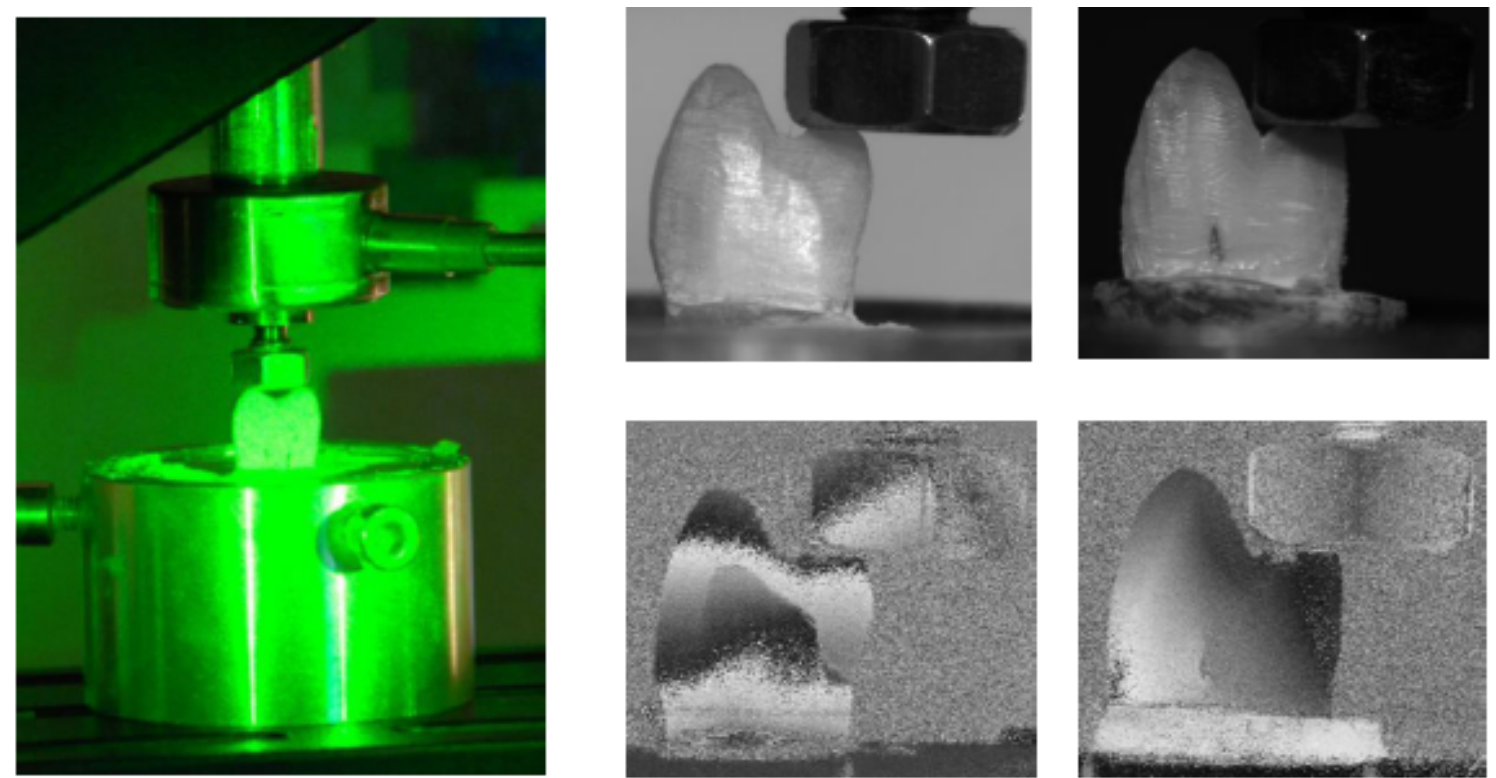

Fig. 5: Laser and white light images (before powdering) and PSDSPI fringes (left, clone; right, natural tooth)

With the new molds, and so avoiding the surrounding glue effect, the realized tests clearly show the enamel crown part is moving differently from the dentine (Fig.5). This difference is clearly delimited by a curve perfectly fitting the dentine-enamel junction (DEJ), as showed on the white light pictures. For the ceramic crown clone, the same kind of shift occurs at the cement junction between the ceramic crown and the dentine ${ }^{6}$.

The loading range applied to the different samples is between $5 \mathrm{~N}$ and $120 \mathrm{~N}$. Clone crown and natural tooth showed differential displacements: about $60 \mathrm{~nm}$ for DEJ and about $100 \mathrm{~nm}$ for the glue joint at the applied loads ( $20 \mathrm{~N}$ and $40 \mathrm{~N})$. Each sample behaves as single solid beyond $120 \mathrm{~N}$.

Around $200 \mathrm{~N}$ some samples have been destroyed due to fragile brittle fracture. This has been confirmed by compression test performed on different testing machine. We used high speed imaging to record this behaviour and also to reach the compression limits of the glue used to fix the samples on the lower jaw.

These measurements show that the natural enamel crown moves independently from dentine. This difference is clearly delimited by a line corresponding to the anatomical location of the dentine-enamel junction (DEJ). For the ceramic clone the same kind of shift occurs at the glue junction of the ceramic crown with the dentine ${ }^{6}$.

Quantitative displacements along the sensitivity vector in the $\mathrm{X}$ direction are represented in figure 6 . The results are presenting two different cases of load. Profiles have been investigated at locations showing differential behaviours. The displacement step is about 50 to $80 \mathrm{~nm}$ for the presented samples. This strengthens the DEJ action as a critical zone 
accommodating the deformation between dentine and enamel. For the clone, the same behaviour and accommodation effect also occurs but for the glue space between the crown clone and the dentine.

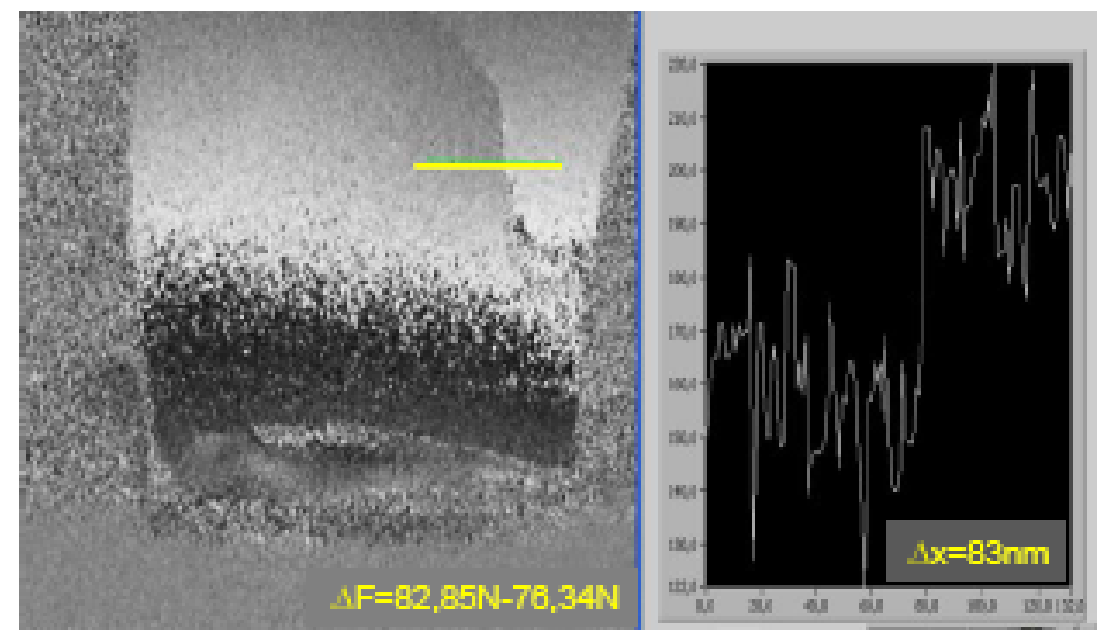

Fig. 6: Displacement map and profile for the clone tooth

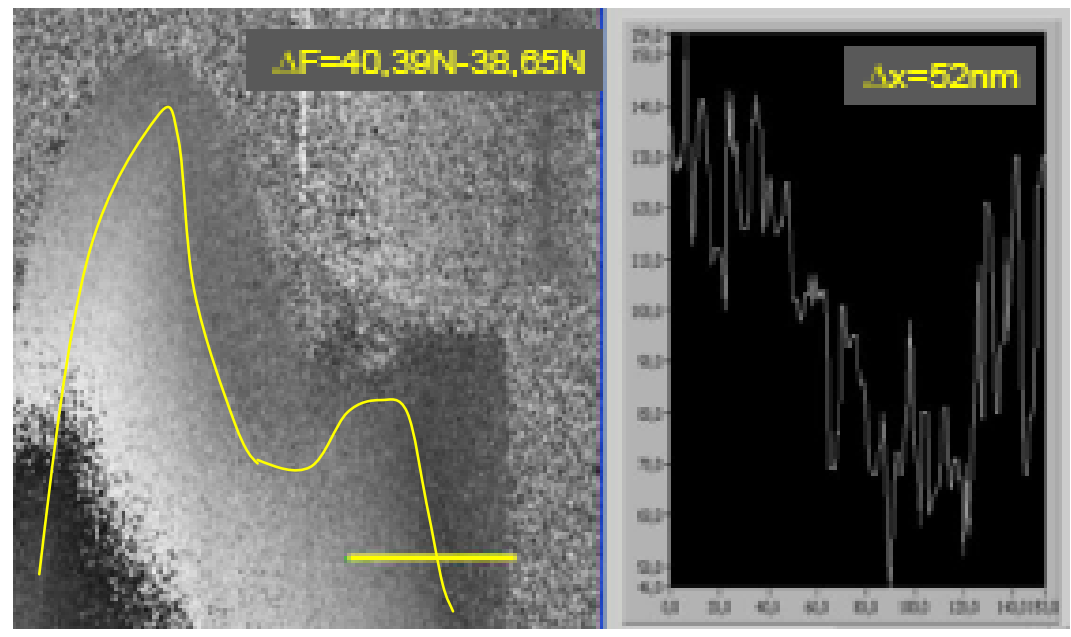

Fig. 7: Displacement map and profile for the natural tooth

\section{CONCLUSION}

This paper deals with the application of phase shifting speckle interferometry to mechanical compression test of tooth. Samples have been realized through CAD-CAM shape duplication to get identical samples. The displacement maps obtained for different loads have shown differential behaviour zones in the X direction. For the natural tooth it is located in the dentine enamel junction whereas for the ceramic crown clone it is located in the cement junction. Both samples present differential displacement of about 50 to 80 nm for loads around $70 \mathrm{~N}$.

The results are really interesting for people from dental research and provide a new way of investigation and of qualification in ceramic crown development.

In further processing, optical displacements will be compared to finite elements analyses of the tooth. Mechanical sensitivity of the tooth surrounding medium will also be examined.

A full 3D measurement will also be done by changing the interferometer sensitivity vectors during the test. 


\section{REFERENCES}

[1] Xu, H H, Smith, D T, Jahanmir, S, Romberg, E, Kelly, J R, Thompson, V P, Rekow, E D, “Indentation damage and mechanical properties of human enamel and dentin", J.Dent.Res., 77,3:472-480, (Mar 1998)

[2] Zaslansky, P, Shahar, P, Barak, M M, Friesem, A A, Weiner, S, "Tooth and bone deformation: structure and material properties by ESPI", Proc. SPIE, 6341:6341091-6341096, (2006)

[3] Barak, M.M. et al., "Enamel dictates whole tooth deformation: A finite element model study validated by a metrology method“, J. Struct. Biol., 2009, in press

[4] Slangen, P, De Veuster, C, Renotte, Y, Berwart, L, Lion, Y, "Computer-aided interferometric measurements of drift and phase shifter calibration for DSPI (Digital Speckle Pattern Interferometry)", Opt.Eng., 34,12:3526-3530, (1995)

[5] Creath, K, Phase-measurement interferometry techniques, Progress in Optics XXVI, Elsevier Science Publisher B.V., Chapt.5, 349-393, (1988)

[6] Fages, M, Cloitre, T, Slangen, P, Corn, S, Cuisinier, F J G, "Speckle Interferometry of Natural and ceramic crown under loading”, Joint Meeting IADR, Barcelona, July 2010 